Wagner, P.; Nagel, K.

\title{
Comparing traffic flow models with different number of "phases"
}

Journal article | Accepted manuscript (Postprint)

This version is available at https://doi.org/10.14279/depositonce-8341

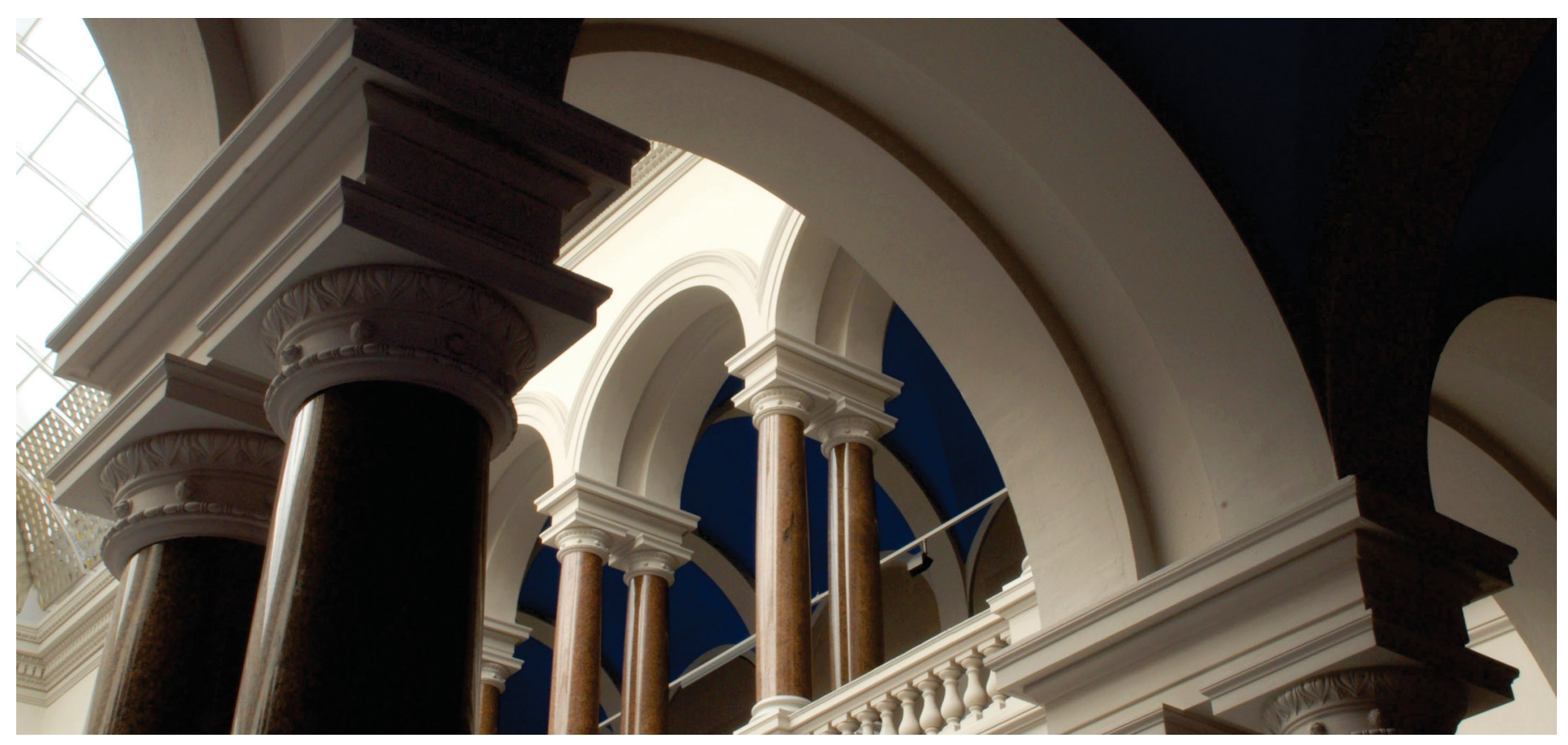

This is a post-peer-review, pre-copyedit version of an article published in The European Physical Journal B. The final authenticated version is available online at: http://dx.doi.org/10.1140/epjb/e2008-00078-0

Wagner, P.; Nagel, K. (2008). Comparing traffic flow models with different number of 'phases'. The European Physical Journal B, 63(3), 315-320. https://doi.org/10.1140/epjb/e2008-00078-0 


\title{
Comparing traffic flow models with different number of "phases"
}

\author{
Peter Wagner ${ }^{1}$ and Kai Nagel ${ }^{2}$ \\ 1 Institute of Transportation Systems \\ German Aerospace Centre, Rutherfordstraße 2, 12489 Berlin \\ peter.wagner at dlr.de \\ 2 Institute for Land and Sea Transport Systems \\ Technical University Berlin, Salzufer 17-19 Sekr. SG12, 10587 Berlin, Germany \\ nagel at vsp.tu-berlin.de
}

\section{Introduction}

Currently, there is an ongoing debate in the traffic flow community concerning the correct empirical and theoretical description of the observations reported in [2]. Mainly three candidates are striving to describe the empirical observations. Following a good tradition, we will call those different approaches hypotheses in the following. Their final goal is to explain (or, a bit more cautious, to describe) the various traffic flow patterns. The different models itself will be described in this text from the perspective of microscopic traffic flow (car-following) models, and we rely on the most simple of those models. These simple models are not capable to deliver a thorough microscopic description of traffic flow (which still needs to be worked out) [4]; this would require to incorporate lane-change phenomena as well as the tactical level of decision making of human drivers $[5,6]$. Nevertheless, it is hoped for that they capture the main macroscopic features, i.e. the patterns of traffic flow observed in reality. For microscopic models, a number of reviews are available, see [7, 8, 9].

Microscopically, car-following is described as an ordinary differential equation (ODE) or one of its discretizations (in time, or in time and space):

$$
\dot{v}=f(g, v, V, \ldots)
$$

Here, $v$ is the speed of the vehicle under consideration, $V$ is the speed of the vehicle in front and $g$ is the net headway to this vehicle. Of course, the model can be stochastic, in which case the equation (1) turns into a stochastic differential equation (SDE). More likely than not, there is a delay of the reaction of the driver to the current state, in which case one ends up with a delay differential equation (DDE). Note, however, that this delay time needs not to be fixed in reality, and when a driver reacts in an anticipatory fashion to the conditions in front, the delay time might even change sign. 
With equation (1), the three different hypotheses about the nature of traffic flow can be discussed in very simple terms. Consider stationary states of equation (1), defined as $\dot{v}=0$. It is plausible, that this can be realized only if $v=V$, with this information the equation

$$
f(g, v, v)=0
$$

can be solved in very general terms. If the equilibrium equation has solutions at all (this is not necessary, it is possible to construct functions $f(g, v, V)$ without this feature), then the stability of those solutions can be discussed.

The simplest case is that all possible solutions $\left(g^{*}, v^{*}\right)$ of $f\left(g^{*}, v^{*}, v^{*}\right)=0$ are stable, and that they organize along a one-dimension curve $v^{*}\left(g^{*}\right)$. This corresponds to a model where the macroscopic behaviour of traffic flow displays just one phase, and especially the equilibrium curve $f\left(g^{*}, v^{*}, v^{*}\right)=0$ of the microscopic model can be transformed into the so called fundamental diagram of the traffic engineers, which relates traffic flow $q=\frac{v^{*}}{g^{*}+\ell}$ with traffic density $k=\frac{1}{g^{*}+\ell}$. Here, the variable $\ell$ is the average generalized length of the vehicles, i.e. the physical length of the vehicle plus the distance a driver holds to the vehicle in front when standing in a jam. Empirically, the fundamental diagram is easily accessible, at least the traffic flow $q$ and the average speed $v$ can be measured directly by double induction loops. Since $q=k v$, under certain circumstances the fundamental diagram in the $(k, q)$-plane can be derived from these data. Upon averaging such a cloud of data into bins along the $k$-axes, a curve $\langle q\rangle(k)(\langle\cdot\rangle$ averages all data-points with the same density $k$ ) can be constructed empirically. It is another discussion, whether this averaging is really sensible from a statistical or a dynamical point of view. Nevertheless, in most cases the curve $\langle q\rangle(k)$ of the fundamental diagram deviates only slightly from a piecewice linear function. We will come back below to microscopic models which have this feature, let us note in passing that the first-order fluid-dynamical Lighthill Whitham Richards theory [14, 15] belongs to this model class.

There is a second class of traffic flow models, where at least some of the solutions of $f\left(g^{*}, v^{*}, v^{*}\right)=0$ are unstable. In this case, the corresponding model displays two phases, a so called free flow phase and a jammed or congested phase. Just as is stated by the theory of first-order phase transitions, there is a mixed regime where a system consists of a mixture of free flow and jammed flow, respectively. To be precise, one should put "phases" and "phase-transitions" in quotation marks, since very often these terms are used in an alarmingly sloppy manner. (Examples are: the order parameter which is linked to any first-order phase transition is not identified at all, its behaviour at the phase transition is not discussed or analyzed, and so on.) Often, a "phase" is just a "regime": having two phases means having (at least) three regimes: (i) the regime where the free flow phase is homogeneous; (ii) the regime where the jammed phase is homogeneous; and (iii) the inhomogeneous regime where the free flow phase and the jammed phase occur simultaneously. 
This is the case for the most simple set-up; in more complicated cases (e.g. for an on-ramp) even richer phase (or regime?) diagrams are possible $[1,3]$.

The final case to be discussed is at the heart of three phase theory. This theory assumes, that the function $f\left(g^{*}, v^{*}, v^{*}\right)=0$ has for a certain range of distances $g^{*} \in\left[g_{1}\left(v^{*}\right), g_{2}\left(v^{*}\right)\right]$ multiple solutions, in fact infinitely many. These points cannot be stable points but are marginally stable, and that means that even a very small amount of noise drives them through the $\left(g^{*}, v^{*}\right)$-plane. Translated back in the fundamental diagram, this means that even in the absence of noise, a cloud of points is existent in the $(k, q)$-plane. To make those models really three-phase, there is another instability point needed: above a certain density, all those points become unstable and then different mixtures of the three phases (free, synchronized, jam) become a possibility. This makes the behaviour of those models very rich, and along with this, very complicated. As with the two-phase models, in more complicated cases a plethora of "phases" (regimes?) is observed in those models.

Note that on a quantitative level the two-phase and the three-phase models display different states ("phases", regimes) which strongly resemble empirically observed patterns of traffic flow.

\section{Models used}

To our knowledge, there is so far not a single model which can be parameterized to yield one, two, or three phase behaviour. There are, however, models which can be parameterized to change between one and two-phase behaviour. Since this contribution is not aiming at the most realistic model, we look for simple possibilities. One the simpler one/two-phase models is the optimal velocity model (OVM) [10], while the simplest known three-phase model is the CA model introduced in [12], named KKW in the following. However, for the scenario below this KKW model turned out to be too simple, since not all parameters could be tweaked to the values needed for this scenario, so a more complex three phase model had to be used [3]. The two models used in this paper are deterministic models, with one exception: an open scenario will be regarded, with a feeding of the models which is a Poissonian source of vehicles. Although it might be very optimistic to assume real traffic to be deterministic, this greatly simplifies matters since the observed patterns are very clear and not covered by noise.

\subsection{OVM}

The OVM model used here is not the one originally introduced in [10], but a slightly modified version of it. Let $x_{n}(t)$ the position of vehicle $n$ at time $t$, and $v_{n}(t)$ its velocity. To stay as simple as possible, consider one lane traffic, so the vehicles are ordered by their positions, $x_{n}(t)<x_{n-1}(t)$. As a further simplification, introduce the net headway between two vehicles as $g_{n}(t):=$ 
$x_{n-1}(t)-x_{n}(t)-\ell$, where $\ell$ is the vehicle length. Again, to simplify matter, all the vehicles have the same length. Then the differential equation of the models reads:

$$
\sigma \dot{v}_{n}(t)=F\left(g_{n}(t)\right)-v_{n}(t)
$$

Here, $\sigma$ is a relaxation time, $F\left(g_{n}(t)\right)$ is the optimal velocity function, which is modelled as piecewise linear [11]:

$$
F(g)=\left\{\begin{array}{lll}
0 & \text { if } & g<g_{1} \\
\frac{g-g_{1}}{\tau} & \text { if } & g_{1} \leq g<g_{2} \\
v_{\max } & \text { if } & g \geq g_{2}
\end{array}\right.
$$

The parameters in this equation are $g_{1}$ the minimum headway (can be zero), the maximum speed $v_{\max }$, the preferred headway $\tau$ of the driver and the maximum look-ahead distance $g_{2}$, which of course is determined by $g_{2}=$ $g_{1}+v_{\max } \tau$.

In all simulations to follow, the model equations will be integrated numerically by the following second order scheme

$$
\begin{aligned}
& v(t+h)=v(t)+\frac{h}{\sigma}(F(g(t))-v(t)), \\
& x(t+h)=x(t)+\frac{h}{2}(v(t+h)+v(t)),
\end{aligned}
$$

where the index has been dropped to improve readability. The parameter $h$ is the step-size, which is usually set to $h=0.2 \mathrm{~s}$. To minimize numerical problems (the model is not crash-free), in any time-step the state of each vehicle is forced to follow the generalized CA crash-free condition, $v<g / h$. In most of the following simulations, this is really needed.

As is well-known, this model possess an equilibrium solution $\dot{v}_{n}(t)=0, \forall n$. It is given by:

$$
v^{*}=F\left(g^{*}\right)=\frac{g^{*}-g_{1}}{\tau}
$$

and it becomes unstable, if the following condition for the putative equilibrium distance $g^{*}$ is fulfilled [10]:

$$
\sigma>\frac{1}{2 F^{\prime}\left(g^{*}\right)}=\frac{\tau}{2}
$$

Provided, of course, that $g_{1}<g^{*}<g_{2}$ is fulfilled, outside of this range the solution to the model is stable.In the simulations below, the parameters of the model are fixed at $g_{1}=0, \ell=6.5 \mathrm{~m}$ and $\tau=1.3 \mathrm{~s}$ with the exception of $\sigma$ which is either $1.0 \mathrm{~s}$ (unstable) or $0.5 \mathrm{~s}$ (stable) to switch from one-phase to two-phase mode. 


\subsection{Kerner's SA (speed adaptation) model (SAM)}

The three-phase model in this field is provided by the recently published speed adaptation model [3], which will be called SAM in the rest of this text. This model is an ODE model, which in its simplest version reads:

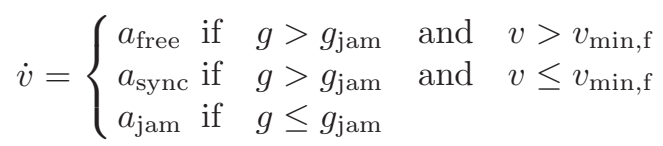

$$
\begin{aligned}
& \dot{x}=v
\end{aligned}
$$

(again the equation without subsript is used, to improve readability) where the different accelerations are defined as:

$$
\begin{aligned}
& a_{\text {free }}=A_{\text {free }}\left(F_{\text {free }}(g)-v\right)+k(v, V)(V-v) \\
& a_{\text {sync }}=A_{\text {syn }}\left(F_{\text {Sync }}(g)-v\right)+k(v, V)(V-v) \\
& a_{\text {jam }}=-k_{\text {jam }} v .
\end{aligned}
$$

with the three remaining functions:

$$
\begin{aligned}
k(v, V) & = \begin{cases}k_{1}(1-\lambda(v))+k_{2} \lambda(v) \text { if } & v \leq V \\
k_{\text {acc }} & \text { else }\end{cases} \\
\lambda(v) & =\frac{1}{1+\exp \left(\left(v / v_{\text {crit }}-1\right) / \epsilon\right)} \\
F_{\text {sync }}(g) & =\frac{g-g_{\text {jam }}}{\tau_{\text {Sync }}} \\
F_{\text {free }}(g) & =v_{\max } \tanh \left(\frac{g}{v_{\max } \tau}\right) .
\end{aligned}
$$

The same parameters as in [3] have been used, with the exception of $v_{\max }=33$ $\mathrm{m} / \mathrm{s}$ which has been set to the same value as for the OV model.

\section{The Leutzbach scenario}

There is a nice example in [13], which will be used in the following to discuss the differences between the different models. A one-lane open road with a stochastic inflow $q$ is considered, which runs from $x=0$ up to $x=20000 \mathrm{~m}$. At the position $x=15000 \mathrm{~m}$ an incident blocks the road completely for 10 minutes $(T=600 \mathrm{~s})$, after that the incident is operated for another $T=600$ seconds at a configurable capacity $q_{\text {out }}$. This is modelled by an adaptive traffic light which switches to green when a time interval $1 / q_{\text {out }}$ has been elapsed since the last switch to green, and stays so until one vehicle has passed it.

Additionally to this description, the boundary conditions need to be taken into account as follows: at the outflow end $(x=20000 \mathrm{~m})$ a completely open 


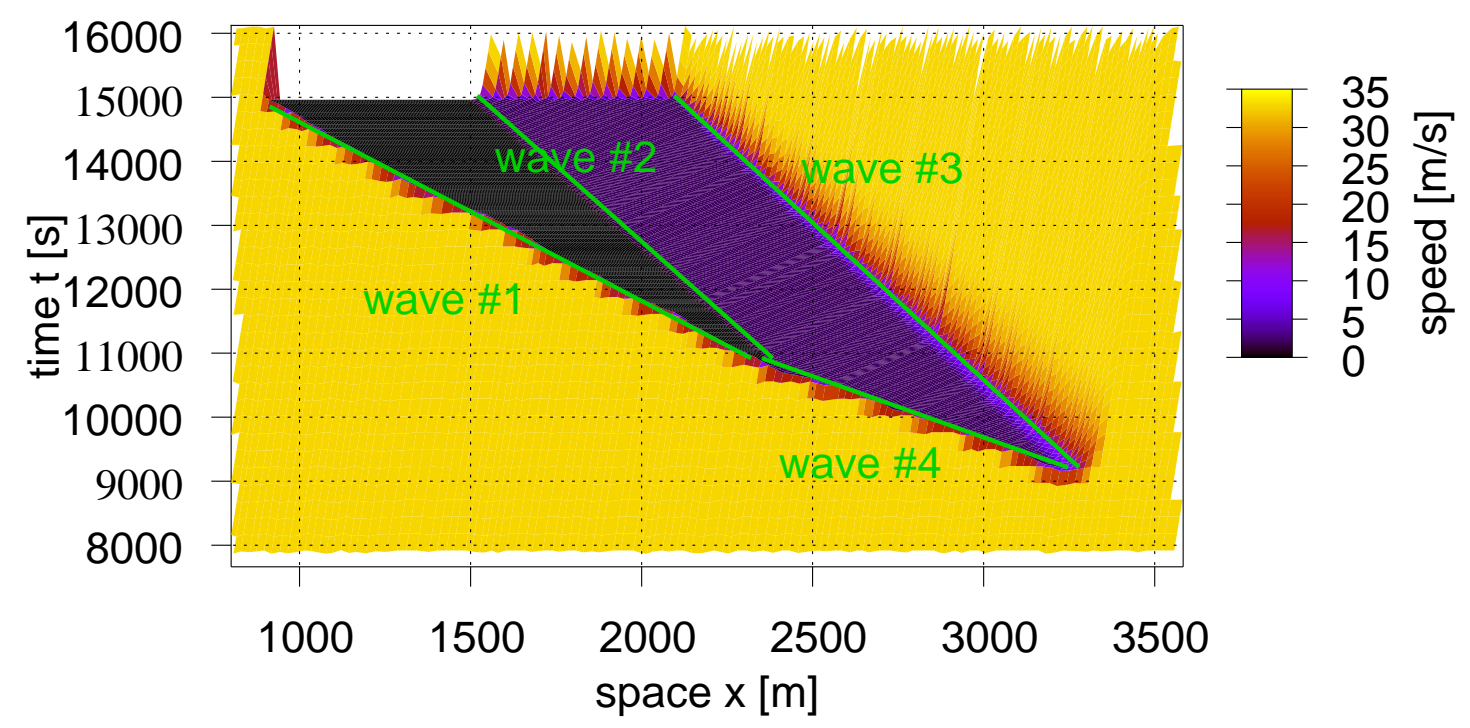

Fig. 1. Space-time diagram of LWR-type of traffic. The shockwaves 1 to 4 are included and labelled in the plot.

boundary is used, i.e. no speed limits nor a capacity constraint is put there. This means, that no external disturbances enter the system. On the inflow end $(x=0 \mathrm{~m})$, vehicles are inserted randomly with a certain rate that corresponds to a the inflow $q$. However, some pre-cautions must be taken to avoid strange phenomena at the inflow end. In principle, vehicles should be inserted roughly with the speed of the vehicle in front $\left(v_{n-1}\right)$ which is already in the system. To do that, one must find a distance $g_{n}$ where $f\left(g_{n}, v_{n}, v_{n-1}\right)=0$, which in case of the OVM model reads $F\left(g_{n}\right)=v_{n-1}$. Basically, this sets the acceleration of the inserted vehicle to zero. This may lead to an insertion point which is outside of the system, $x_{n}<0$. If the last vehicle is already outside of the system, i.e. if $x_{n-1}<0$, then no new vehicle is allowed to enter the system. This effectively shuts down the inflow, although it is of course not a realistic description of real traffic, it will be used here for convenience.

\subsection{Results}

The scenario described above generates five shock-waves, four of them are important in what follows and are drawn in Fig. 1. As could be seen in Fig. 1, the simulation of the OVM model described above reproduces almost exactly the simulation results in line with LWR theory - for an appropriate choice of the parameters, of course. The first wave (named wave \#1 in Fig. 1) starts at the beginning of the blockade, the second wave when the blockade is lifted partially, and the third wave starts when the freeway is free again. The collision of wave \#1 with wave \#2 produces a new wave \#4, and all patterns vanish 
when this wave \#4 finally collides with wave \#3. Of course, for very small inflow, i.e. if $q<q_{\text {out }}$ just two waves remain.

Just to make it clear: LWR and the OVM with the set of parameters chosen for Fig. 1 is what physicists call a one-phase model.

The following set of figures demonstrates what happen upon the transition from a one-phase model to a two-phase model. The OVM display this transition if the relaxation time $\sigma$ gets large enough, i.e. if $\sigma<\tau / 2$. As has been detailed above, this means microscopically that the solution $\dot{v}_{n}=0$ becomes unstable. The following plot displays the difference between a parameter setting where OVM is one-phase and two-phase, respectively.
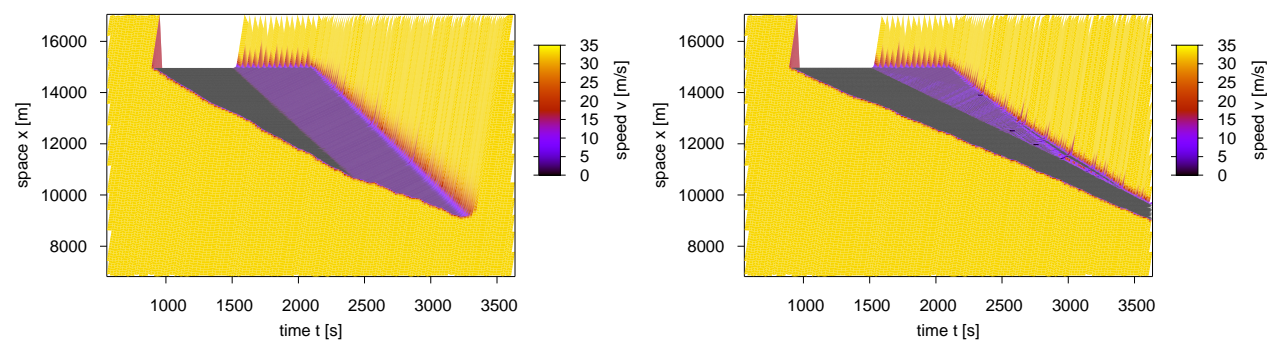

Fig. 2. Space-time diagram, left panel is LWR similar with the relevant parameters given by $q_{\text {in }}=0.5, \quad \sigma=0.5, \quad q_{\text {out }}=0.25$; the right plot is with $\sigma=1.0$, in the two phase regime. Both plots look similar, because the inflow is below a critical value where both models show marked differences. It is, however, difficult with plain LWR to construct a convincing model where the wave front between a low and a medium density area runs backward faster than the wave front between a medium and a high density area, as it is the case in the plot on the right.

First of all, if the inflow is too small, there is no marked difference in this scenario between a one-phase and a two-phase model (see Fig. 2). It is, however, difficult with plain LWR to construct a convincing model where the wave front between a low and a medium density area runs backward faster than the wave front between a medium and a high density area, as it is the case in the plot on the right. For this, one would need a fundamental diagram that is convex (i.e. curved upward) on the congested branch (i.e. for densities above the density of maximum flow). Such a fundamental diagram has, however, the property that the upstream front of a jam (i.e. the deceleration wave) spreads out over time - a feature that does not seem plausible with respect to reality. This is discussed in more detail in [16].

Upon increasing the inflow above a value $q>q_{c}$, where $q_{c}$ is the outflow from a stable traffic jam (which is a number which is simply not available in simple LWR), things start to change. This can be seen in Fig. 3. There are two features which uniquely discern a two-phase from a one-phase model. First, the 

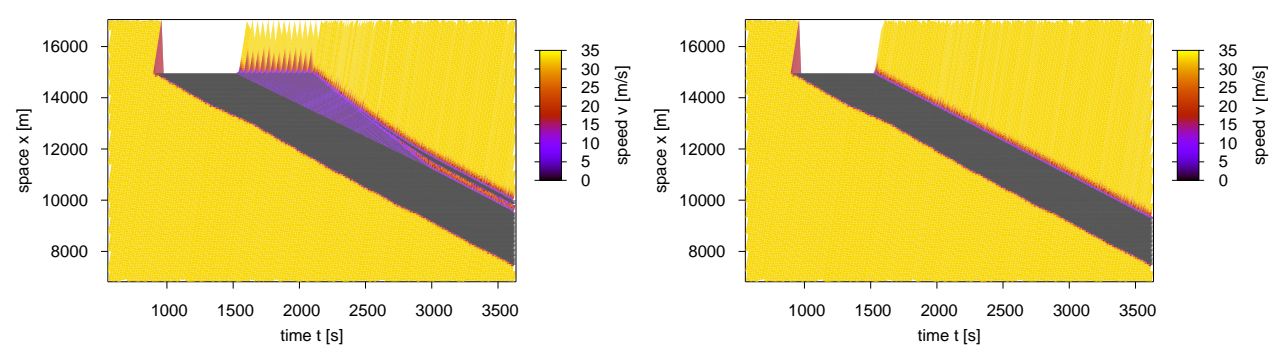

Fig. 3. Space-time diagram, this time with $\sigma=1.0$. Left panel is with large inflow, but small outflow from the incident $\left(q_{\text {in }}=0.7, \quad q_{\text {out }}=0.25\right)$; in this case, a different pattern is observed, because the large inflow stabilizes the jam. By increasing the outflow from the incident above the outflow from the jam $\left(q_{\text {out }}=0.5\right)$, all relevant patterns with the exception of the jam vanish.

large inflow can completely stabilize the jam. This means that the upstream front of the jam runs faster upstream than the downstream front. While there is just one single inflow in LWR where this can (marginally) happen, in the case of a two-phase model there is a whole range of inflows $q_{c}<q<q_{\max }$, with $q_{\max }$ the maximum possible flow that can be reached by the model or in reality. Note, that this fact makes it very likely to observe such a phenomenon with a two-phase model, while it is not very likely for a one-phase model. The second feature is that there is a region in parameter space, where the congestion caused by the partially lifted incident is completely suppressed, see Fig. 3 (right) for an example. Of course, this may happen twice, i.e. for a very small inflow, too, but especially the pattern observed in Fig. 3 (right), where $q_{\text {out }}$ is still macroscopically smaller than $q_{i n}$, is impossible for a onephase model.

As could be expected, an even different picture is observed from the three phase model, see Fig. 4 for an example. When the outflow from the partial blockade is small, then directly upstream of the incident a homogeneous region of traffic flow can be observed. This breaks down into a sequence of small jams. What changes upon increasing of $q_{\text {out }}$ is the frequency between these jam waves, it gets smaller until they finally vanish into a similar structure as in the right part of Fig. 3. So, in this congested region there is an additional substructure which is not apparent in two-phase models. Further analysis is needed to confirm the detailed structure of this congested phase.

\section{Discussion}

With the help of a fairly simple scenario, the differences between one-phase, two-phase, and three-phase models have been demonstrated. The idea behind 

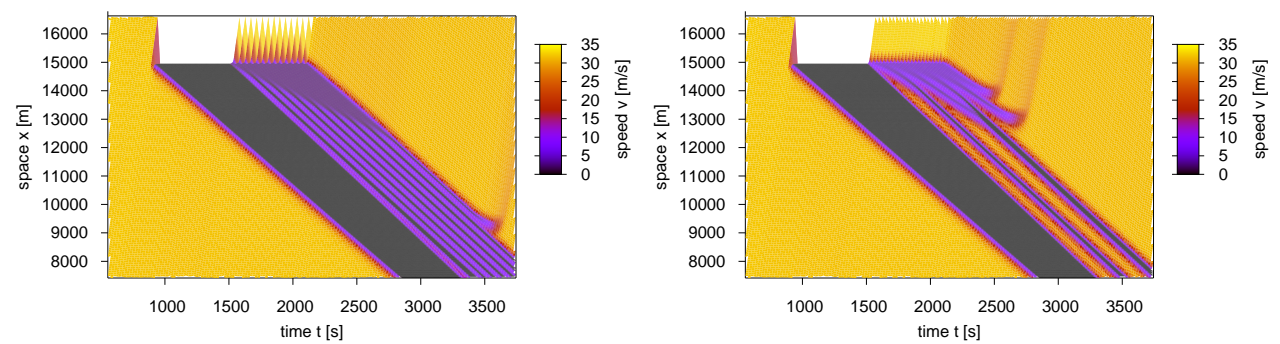

Fig. 4. Space-time diagram, this time for the SAM model. The behaviour of this model is different from the one- and two-phase models. The parameters (which were not so easy to tweak) are $q_{\text {in }}=0.5$ for both figures, and $q_{\text {out }}=0.22$ in the left and $q_{\text {out }}=0.36$ in the right plot. The parameters of the model had been set to the values in $[3]$

this simple approach is to better understand which type of patterns is generated by which model class. Furthermore, similar approaches might help in analyzing empirical patterns. E.g., there are clear differences in this simple scenario between the different models, which could be observed empirically and can therefore help to decide what reality all about is. Note, however, that it might happen that reality is more colorful than the simple theories presented here: given a different driver collective on a different day, they might as well "decide" to organize as a one, two, three-phase traffic flow, just to make life miserable for traffic flow theoreticians.

Be that as it is, the next step to be taken is to quantitatively (and not only qualitatively) test these and other theories with real data. The most promising candidate to do so is unfortunately not the scenario investigated here, because it might be impossible to obtain enough examples to even draw statistically meaningful answers, but the on-ramp scenario which is used in almost all the publications related to the different phases of traffic flow.

\section{References}

1. M. Treiber, A. Hennecke, and D. Helbing Physical Review E 62, 1805-1824 (2000).

2. B. S. Kerner, Phys Rev E 65:046138 (2002).

3. B. S. Kerner, S. L. Klenov J. Phys. A: Math. Gen. 39 1775-1809 (2006), doi:10.1088/0305-4470/39/8/002

4. P. Wagner, Europ. J. Phys. B 52, 427 (2006), DOI: 10.1140/epjb/e2006-00300-1

5. T. Toledo, H. N. Koutsopoulos, and M. Ben-Akiva Integrated Driving Behaviour Modeling, to be published in Transp. Res. C (2007).

6. P. Vortisch, private communication (2007).

7. D. Chowdhury, L. Santen, and A. Schadschneider, Phys. Rep. 329, 199 (2000). 
8. D. Helbing, Rev. Mod. Phys. 73, 1067 (2001).

9. K. Nagel, P. Wagner, and R. Woesler, Oper. Res. 511 (2003).

10. M. Bando, K. Hasebe, A. Nakayama, A. Shibata, and Y. Sugiyama, Physical Review E, 511035 (1995).

11. K. Nakanishi, K. Itoh, Y. Igarashi, M. Bando Phys. Rev. E 55, 6519 (1997)

12. B. S. Kerner, S. L. Klenov, and D. W. Wolf, J. Phys. A: Math. Gen., 35, 9971(2002)

13. W. Leutzbach,Introduction to the Theory of Traffic, Springer, Berlin, (1988).

14. M. J. Lighthill and J. B. Whitham, Proceedings of the Royal Society A $\mathbf{2 2 9}$, 281 (1955).

15. P. I. Richards, Operations Research, 4, 42 (1956)

16. K. Nagel, P. Nelson: A critical comparison of the kinematic-wave model with observational data. In: Transportation and Traffic Theory - Flow, Dynamics, and Human Interaction (Proc. 16th ISTTT), ed by H. Mahmassani (Elsevier, 2005), pp 145-164 